\title{
自动化技术在机械设计与制造的应用
}

\author{
王晓楠 \\ 天津龙创恒盛实业有限公司，天津 301600
}

[摘要]近年来, 在各方面利好因素的影响下, 使得我国综合国力得到显著的提升, 从而推动了我国社会经济整体水平的不断 提升。在这种发展趋势下，为我国机械设计制造行业的发展创造了良好的条件，科技的不断发展使得机械设计制造行业的生 产模式出现了显著的变化, 这样也为整个机械制造行业提出了更高的要求。在机械设计制造行业中切实的引用自动化技术, 以互联网和计算机技术为基础，大范围的运用最前沿科学技术，推动机械设计制造行业的稳步发展壮大。 [关键词]机械设计制造; 自动化技术; 应用分析

DOI：10.33142/ec.v3i5.1918 中图分类号：TH122;TH164 文献标识码：A

\section{Application of Automation Technology in Mechanical Design and Manufacturing}

\author{
WANG Xiaonan
}

Tianjin Longchuang Hengsheng Industrial Co., Ltd., Tianjin, 301600, China

\begin{abstract}
In recent years, under the influence of various favorable factors, China's comprehensive national strength has been significantly improved, thus promoting the continuous improvement of the overall level of China's social economy. In this development trend, good conditions have been created for the development of China's mechanical design and manufacturing industry. With the continuous development of science and technology, the production mode of mechanical design and manufacturing industry has changed significantly, which also puts forward higher requirements for the whole mechanical manufacturing industry. In the mechanical design and manufacturing industry, automation technology is effectively used. Based on the Internet and computer technology, the most cutting-edge science and technology are widely used to promote the steady development and growth of the mechanical design and manufacturing industry.
\end{abstract}

Keywords: mechanical design and manufacturing; automation technology; application analysis

引言

经过实践调查我们发现，在我国工业生产行业之中，切实的引用自动化设备能够有效的提升生产的效率和水平， 并且促使工作人员从巨大的工作量中摆脱出来，实现了控制人工成本的目的，自动化设备逐渐的成为了当前信息时代 中工业高效生产中的主要部分。这就充分的说明了, 自动化技术水平与整个制造行业的发展存在密切的关联, 所以围 绕自动化技术在机械设计制造中的实践运用展开全面分析研究其意义是十分巨大的。

\section{1 概述机械自动化及组成部分}

\section{1 机械自动化的概述}

机械自动化其实质就是机械在完全摆脱人为操作的情况下依据前期设定的流程进行各项工作的操作。机械自动化 与自动化技术二者之间存在一定的类似性, 但是二者却属于两个不同的范畴。机械自动化其实质就是运用机械设备来 落实各项生产工作，其与以往陈旧的生产模式存在明显的区别。就生产制造企业来说，控制生产成本、提升生产效率 始终都是企业发展的核心目标。但是就现如今大部分制造企业实际情况来说, 大部分的生产机械设备综合性能水平较 为落后, 并且操作工作具有一定的复杂性。通常的时候, 往往需要多个工人协同完成某个设备的操作, 工作效率较低, 并且生产中涉及到的人工成本较多。

\section{2 机械自动化技术的组成}

\section{2 .1 传感单元}

这是机械自动化技术中最为基础的一部分单元，主要功能在于检测整个系统的工作过程。

1.2. 2 作用单元

这个单元的作用在于明确定位整个系统的能力, 也是整个自动化系统之中的能量施加单位。 


\subsection{3 程序单元}

这块单元的作用在于施加指令, 来有效决定整个系统的操作单元。

1.2 .4 控制单元

这部分的作用在于对传感单元传送过来的信息数据进行定位以及检测, 同时对这部分信息数据进行传输。换言之, 也是整个系统之中的后勤保障部门 ${ }^{[1]}$ 。

\section{2 .5 制定单元}

该部分的功能在于对传感单元传输过来的信号信息进行分析，也是整个自动化系统之中的关键单元。

\section{2 自动化技术在机械设计制造中应用的必要性}

\section{1 可提高生产效率}

就当下我国社会经济发展状况来说, 整体发展速度不断的提升, 从而推动了整个机械设计制造行业的稳步发展, 促使整个行业市场竞争形势越发的严峻, 机械设计制造企业要想在如此严峻的竞争形势下长期处在不败的境地, 最为 重要的就是需要从各个角度入手来提升自身的综合实力。科学技术的飞速发展, 使得自动化技术水平得到了显著的提 升, 并被人们大范围的引用到了机械设计制造行业之中, 取得了十分显著的成效。运用自动化和信息化技术对机械设 计制造体系进行不断的优化完善, 能够促使企业获取更加丰厚的经济和社会效益 ${ }^{[2]}$ 。

\section{2 可降低生产成本}

长时间以来, 我国机械制造行业往往都是利用人工操作的方式来实施产品的设计和制造的, 从而使得机械制造企 业生产工作需要大量的人工成本。在自动化技术快速发展的推动下, 人们将自动化技术切实的引用到机械制造生产环 节之中从而实现的自动化生产替代人工操作的目的, 有效的缩减了生产工作所需要的人工成本, 并且对于推动产品生 产工序的自动化和规模化起到了积极的影响作用。尽管机械制造企业在引入自动化生产技术和生产机械的过程中需要 投入较多的成本, 并且要需要大量的专业人员对设备进行安装调试和维护工作, 但是自动化技术所创造的长远经济效 益是以往落后的人工生产所不能及的。

\section{3 有助于实现精准化生产}

在最近的几年时间里, 我国科学技术整体水平得到了巨大的发展, 从而推动了整个机械设计制造行业朝着智能化 和自动化方向发展。在机械设计制造企业中切实的引用自动化技术, 需要诸多高精度机械设备的辅助, 如果设备零部 件的精度较差, 那么必然会对产品设计和制造造成诸多的限制, 所以在引用自动化技术的同时还需要运用电子设备来 完成对产品的设计, 之后设计工作人员需要综合实际情况和需要对产品的设计图进行完善和优化, 从而从根本上确保 产品的设计效果和产品质量能够达到既定的标准 ${ }^{[3]}$ 。

\section{4 有助于充分利用资源}

在机械设计工序之中切实的引入自动化技术, 能够有效的提升各类生产资源的利用效率, 因为机械产品设计与制 造环节中对设备的精度的要求较高, 所以可以将具有一定质量问题的产品进行二次利用进行生产, 从而有效的提升资 源的利用效率。其次, 在实践中引用自动化技术, 能够保证机械设计与制造实现多样化, 可以针对不同种类的材料进 行专门的设计, 从而保证设计结果的可行性 ${ }^{[4]}$ 。

\section{3 自动化技术在我国机械设计制造中的应用}

自动化技术牵涉到的层面较多, 并且具有良好的适用性, 能够被引用到各种不同需求的生产之中。就生产设备实 际情况来说, 当前我国引用在生产之中的高精度生产设备大部分还是依赖进口或者是组装, 被运用在生产关键核心位 置的数控系统通常都是西门子、三菱、法纳克等国外数控系统, 当前我国自主研发并被运用到一线生产系统之中的数 控系统较少。其次, 就机械设计制造行业内的自动化管理工作实际情况来说, 大部分的经济水平较高的国家都已经能 够自行研发自动化管理系统以及信息化管理系统, 并且在实践运用中取得了良好的成绩。但是我国大部分机械设备制 造企业在自动化管理工作方面还没有完全的摆脱人工管理模式的限制, 人工操作在实际机械设计制造行业生产中还是 非常重要的。为了迎合整个行业的发展趋势, 部分企业正在积极的进行转型, 并且在大范围的引入自动化设备。其次, 大量的新型自动化设备也被切实的引用到了实践生产之中, 诸如: 自动上卸料设备、自动装加工艺设备、在线工艺检 测设备等，从而有效的提升了企业整体生产水平，为机械制造行业的稳步持续发展创造了良好的基础。 


\section{4 自动化技术在机械制造中的}

\section{1 集成化应用}

机械自动化核心发展方向就是集成技术。这项技术其实质就是借助专业工程理论来对信息技术进行规范指导, 并 且综合各方面实际情况来对生产工作进行优化完善, 促进自动化生产效率的提升。机械自动化能够将机械制造行业中 涉及到的各种关键要素以及管理活动融合在一起, 从而实现以人为本的生产理念。与企业生产运营工作十分类似, 如 果可以利用有效的方法将生产活动于企业运用管理工作综合在一起, 那么在促进企业生产效率方面能够起到积极的影 响作用 ${ }^{[5]}$ 。在企业生产过程中, 工作人员需要对生产过程中涉及到的各个工序进行综合分析研究, 并对设备运行中牵涉 到的所有重要参数和性能指标加以全面考察, 将各项信息数据进行详细的记录, 这样能够为后续的设备维保工作的开 展创造良好的基础。只有切实的落实实践工作, 并保证数据信息的准确性和全面性, 才能促进机械制造行业的稳定健 康发展。其次, 机械生产工作具有较强的复杂性, 在实际生产过程中需要综合各方面因素加以全面考虑, 不但需要从 各个细节入手进行分析, 并且最后还需要加以整合考虑, 不然极易对设备正常运转造成一定的阻碍。所以我们需要综 合生产产品实际情况这对生产系统中各个部件参数进行调整, 并对生产中遇到的各类问题加以切实的解决。其次, 需 要针对生产设备运行需要支出的各项费用进行全面的管控, 综合设备的使用环境, 利用专业的方法来获取各项数据进 行数学模型的创建。

\section{2 数控技术}

在社会经济水平大幅度提升的带动下, 推动了机械生产行业的飞速发展, 各个行业对机械生产产品的需求量也在 不断的提升。以往老旧模式的人工操作生产模式很显然已经无法满足市场的需求了, 并且在保证产品质量方面也不具 备良好的作用, 对于各类资源的需求量较大。而将自动化技术切实的引用到机械生产之中, 能够有效的解决以上问题。 数控技术是自动化技术之中最为关键的一项专业技术, 其实质就是借助电子设备对机械设备进行操控, 技术工作人员 只需要对设备的运行流程进行前期的设定, 设备就可以自行完成生产工作, 有效的提升生产的效率, 尽可能的提升各 类资源的使用效率 ${ }^{[6]}$ 。

\section{3 虚拟技术}

虚拟技术是人工智能技术、电子信息技术以及多媒体技术的综合体, 其主要原理就是利用方针以及模拟技术来讲 各类技术引用到机械自动化生产之中。将这项技术引用到机械自动化生产之中, 在遇到各类生产问题的时候, 借助虚 拟技术能够更加高效的判断出引发生产问题的根源, 从而在短时间内加以高效的解决, 确保各项生产活动能够有序的 开展。但是就现如今实际情况来说, 虚拟技术整体水平并没有达到完善的状态, 其中存在诸多的问题需要我们进一步 的加以解决。

\section{4 柔性自动化应用}

机械制造行业要想保证稳定持续发展, 最为关键的就是需要彻底的扭转以往的落后观念, 综合整个行业的发展趋 势以及市场需要, 针对机械产品生产和制造工序进行优化完善, 从而实现柔化发展的目的。柔性自动化技术, 其实质 是将信息控制系统、物料运输系统以及数字化控制系统融合在一起的有机整体，能够实现自动匹配生产。

\section{5 结语}

总的来说, 在整个机械设计制造行业之中, 自动化技术的引用不但能够有效的提升生产效率、控制生产成本, 并 且能够最大限度的将各类资源的作用施展出来，为整个机械设计制造行业的稳定健康发展打下坚实的基础。

\section{[参考文献]}

[1]孟辛酉.自动化技术在机械设计制造中的运用 [J].科技风,2020(12): 179-189.

[2]崔跃峰.浅谈自动化技术在农业机械制造中的应用 [J].农业开发与装备, 2020(03): 36-34.

[3]王军.自动化技术在机械设计制造中的新应用 [J].中国科技信息, 2020(06): 60-61.

[4]刘洪波,孙晓金.自动化技术在机械设计与制造中的应用 [J]. 南方农机, 2020,51(04): 158 .

[5]喻天旭.自动化技术在机械设计与制造中的应用研究 [J]. 科技风, 2020(03) : 168 .

[6]易寒,许林杰, 居锋. 自动化技术在机械设计中的应用 [J]. 内燃机与配件, 2020 (02) : 242-243.

作者简介: 王晓楠 (1980-), 男, 总经理, 本科学历, 毕业院校: 河北工业大学, 机械设计制造及自动化专业, 工程 师、产品负责人。 